\title{
Does cytoreduction surgery and hyperthermic intrathoracic chemotherapy prolong survival in patients with NO-N1 nonsmall cell lung cancer and malignant pleural effusion?
}

\author{
Marcello Migliore (1) and Marco Nardini \\ Affiliation: Dipartimento di Specialita Medico-Chirurgiche, Universita degli Studi di Catania, Catania, Italy.
}

Correspondence: Marcello Migliore, Dipartimento di Specialita Medico-Chirurgiche, Universita degli Studi di Catania, Via S. Sofia 78, 95125 Catania, Italy. E-mail: mmigliordunict.it

@ERSpublications

Patients with pleural effusion and lung cancer have a poor survival (3-12 months). Debulking surgery and HITHOC shows $62 \%$ survival at 1 year and $28.5 \%$ at 2 years. Evaluation of this promising novel therapeutic approach in the form of an RCT is mandatory. http://bit.ly/2WPbCXS

Cite this article as: Migliore M, Nardini M. Does cytoreduction surgery and hyperthermic intrathoracic chemotherapy prolong survival in patients with N0-N1 nonsmall cell lung cancer and malignant pleural effusion? Eur Respir Rev 2019; 28: 190018 [https://doi.org/10.1183/16000617.0018-2019].

\section{ABSTRACT}

Introduction: Debulking surgery and hyperthermic intrathoracic chemotherapy (HITHOC) has been successfully used in the treatment of thoracic tumours. Few authors report on the feasibility of its use in patients with lung cancer and malignant pleural effusion. The aim of this study was to evaluate the efficacy and results of debulking surgery and HITHOC in the treatment of selected patients with nonsmall cell lung cancer (NSCLC) and malignant pleural effusion.

Methods: A systematic review was conducted in MEDLINE in accordance with PRISMA guidelines. The word search included: "hyperthermic intrathoracic chemotherapy and/or HITHOC or hyperthermic intrapleural". Inclusion criteria were only those studies reporting a sufficient amount of data on HITHOC and surgery for lung cancer. Single case reports and review articles were excluded.

Results: 20 articles were selected as they related to the topic of HITHOC and lung cancer. Most were from China $(n=8)$ and Japan $(n=6)$. Only four out of the 20 articles had sufficient data for this review. In total, data for 21 patients were collected. Debulking surgery ranged from wedge resection to pneumonectomy and pleurectomy. Mean survival was 27 months and median survival was 18 months (range 1-74 months). 13 patients out of $21(62 \%)$ were alive at 1 year and six (28.5\%) were alive at 2 years. 10 patients were still alive at the time of the respective publication in the 21 patients included. Systemic toxicity and treatmentrelated mortality were nil. There were insufficient data to perform a meta-analysis.

Conclusion: Although reported survival in this systematic review is encouraging, available evidence concerning debulking surgery and HITHOC in N0-N1 NSCLC with malignant pleural effusion is weak. Better evidence in the form of a randomised controlled trial is mandatory.

\footnotetext{
This study is registered at PROSPERO with identifier number CRD42018102155.

Provenance: Submitted article, peer reviewed

Received: 26 Feb 2019 | Accepted after revision: 03 June 2019

Copyright $\odot$ ERS 2019. This article is open access and distributed under the terms of the Creative Commons Attribution Non-Commercial Licence 4.0.
} 


\section{Introduction}

Although more than 100 years have elapsed since the first reports on lung cancer [1], recent studies confirm that long-term survival in patients with nonsmall cell lung cancer (NSCLC) is still unsatisfactory. This is mainly due to the late diagnosis and dismal survival of inoperable patients with advanced disease, who represent $70-75 \%$ of the entire group of lung cancer patients.

Surgery has the main role only in early-stage NSCLC, while nonsurgical treatments are recommended in patients with stage IV. We believe that in order to prolong survival of the entire group of patients affected by NSCLC, great efforts should be made to prolong survival in the inoperable patients.

For this reason, on the basis of our recent experience with cytoreductive surgery and hyperthermic intrathoracic chemotherapy (HITHOC) for mesothelioma and malignant pleural effusion $[2,3]$, and some recent publications reporting good survival in selected patients with stage IVA lung cancer [4-6], we strongly believe that not all patients with stage IVA (M1a) NSCLC are the same and that longer survival can be obtained in those with disease confined in the hemithorax, and therefore a "new" form of precision (individualised) surgery should be explored as part of a multidisciplinary treatment modality. ZHou et al. [7] performed a meta-analysis on the effect of debulking surgery and HITHOC on malignant pleural effusion for various primary cancers. They showed that patients who received HITHOC had a significantly longer survival. ZHOU et al. [7] concluded that HITHOC is safe, effective and increases the survival rate.

We performed a systematic review with the aim to evaluate the efficacy and preliminary results of debulking surgery and HITHOC in the treatment of patients with malignant pleural effusion (M1a) and NSCLC confined in the hemithorax (N0-N1).

\section{Materials and methods}

A systematic review of the literature was conducted in accordance with PRISMA guidelines and the review was registered in the PROSPERO registry of systematic reviews with identifier CRD42018102155. An effort was made to find all publications reporting patients who underwent debulking surgery (pleurectomy, decortications and lung resection), in combination with HITHOC, for advanced stage NSCLC. The search was performed in MEDLINE up to April 30, 2018. As HITHOC is not a common procedure, the key words used in the search were necessarily varied, and included: "hyperthermic intrathoracic chemotherapy and/or HITHOC or hyperthermic intrapleural" in the title and in the abstract.

After screening for duplicates, only articles in English reporting the type of tumour, type of approach, type of resection, survival in months for every single patient and clinical results were included. Unpublished material, congress abstracts and proceedings were not considered. Reviews were also excluded.

The results of the search were screened and reviewed by both of the current authors individually and the results were compared. There were no disagreements between the two authors. Due to the small number of patients and the different outcomes measured, a meta-analysis of the data could not be performed, and therefore this review is limited to the description of the included studies and their results.

\section{Results}

The PRISMA flowchart of the final article selection process is shown in figure 1 . In the title, the words "hyperthermic intrapleural" were present in 168 articles, "hyperthermic intrathoracic chemotherapy" in 85 articles and "HITHOC" in 20 articles.

FIGURE 1 PRISMA flowchart of article selection. HITHOC: hyperthermic intrathoracic chemotherapy.

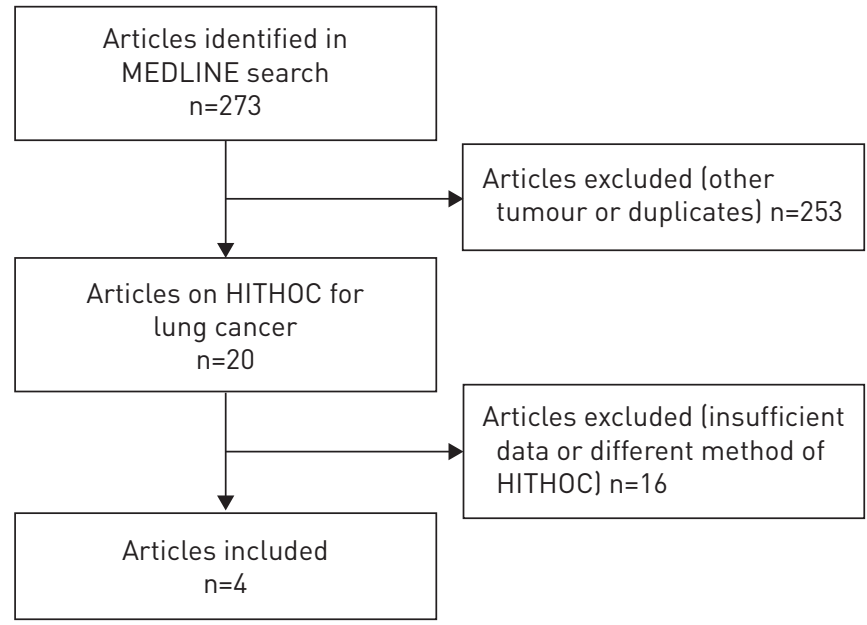


TABLE 1 Evidence table: histopathology, type of surgery and survival data in 21 patients who underwent cytoreductive surgery and hyperthermic intrathoracic chemotherapy (HITHOC)

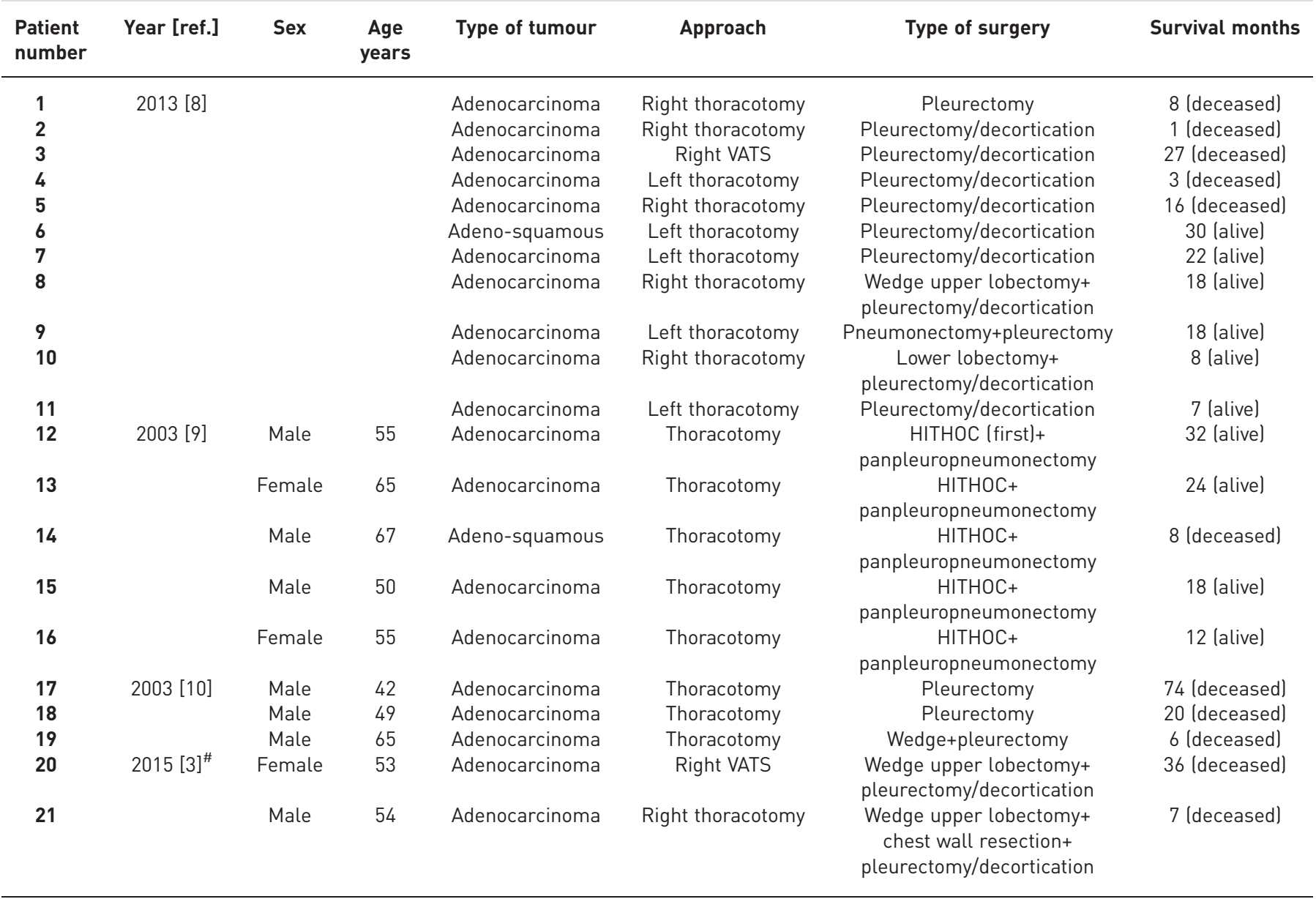

VATS: video-assisted thoracoscopic surgery. ${ }^{\text {\# }}$ : our experience updated from [7].

As shown in figure 1, 20 out of 273 articles were selected as they related to the topic of HITHOC and lung cancer: $n=8$ from China, $n=6$ from Japan, $n=2$ from Korea, $n=1$ from France, $n=1$ from Italy, $n=1$ from Israel and $\mathrm{n}=1$ from Turkey. Of these 20 articles, only four articles with a total of 21 patients had sufficient data for this review (table 1) [3, 8-10]. All 21 patients had undergone debulking surgery and HITHOC. Data was available on age and sex in 10 patients: seven males and three females with a mean age of 55.5 years (range 42-67 years). In the case of positron emission tomography (PET)-positive N2 or N3, endobronchial ultrasound (EBUS) and/or video-mediastinoscopy was performed to guarantee that no patient was denied surgery because of a false-positive computed tomography (CT) or PET scan. Debulking surgery ranged from wedge resection to pneumonectomy and pleurectomy. HITHOC was performed in all patients for $1 \mathrm{~h}$.

Mean survival was 27 months and median survival was 18 months (range 1-74 months). 13 out of the 21 patients $(62 \%)$ were alive at 1 year. Six patients $(28.5 \%)$ were alive at 2 years. 10 patients were still alive at the time of the respective publication in the 21 patients included (figure 2). Systemic toxicity and treatment-related mortality were nil. There were insufficient data to perform a meta-analysis.

\section{Discussion}

It is known that drug infusion into the pleural cavity leads to direct exposure of tumour cells lining the surface of the pleural cavity. Systemic absorption from the pleural cavity is lower when compared with absorption from the peritoneal cavity during intraperitoneal hyperthermic chemotherapy procedures [11]. The hyperthermia itself has a toxic effect on malignant cells [12] and modifies the toxicity of the chemotherapeutic agent [13]. However, systemic concentrations remain below toxic levels due to the limited absorption of the drug from the cavity. By attempting to "sterilise" the microscopic residual disease 
FIGURE 2 Kaplan-Meier survival curve with $95 \%$ confidence intervals (shaded area) for stage IVA (M1a) pleural effusion.

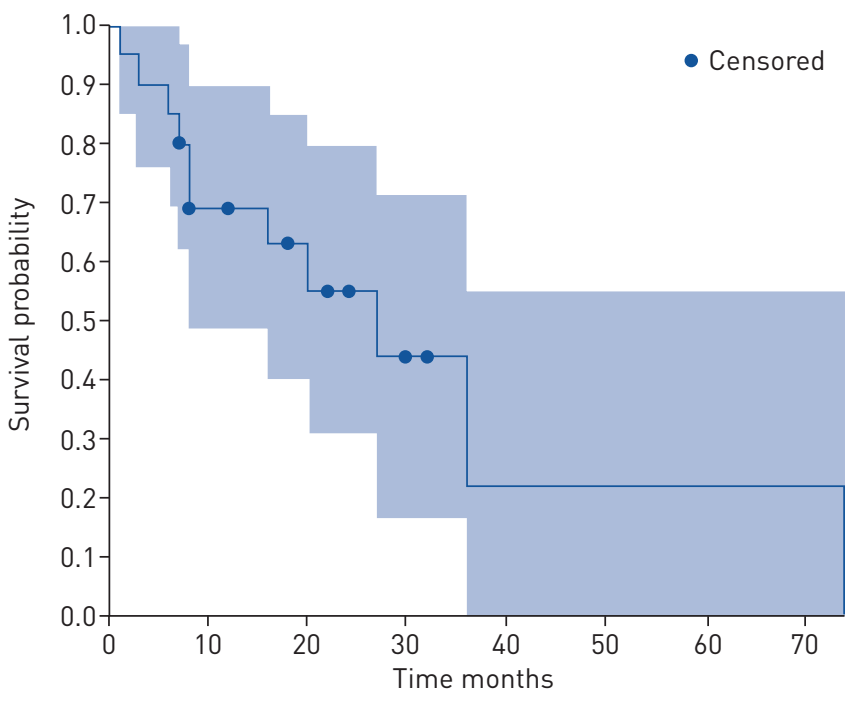

after surgical resection, HITHOC has been successfully used in the treatment of some thoracic tumours such as malignant pleural mesothelioma and thymomas [14-16]. Moreover, a recent well-designed study demonstrated that hyperthermia together with cisplatin is an effective treatment for epidermal growth factor receptor (EGFR) kinase domain mutation-positive lung cancer patients [17]. The authors of the study confirmed that hyperthermia and cisplatin, synergistically, downregulated the EGFR protein level, leading to quenching of the signal from EGFR and induction of apoptosis.

For the same reasons, the hypothesis that cytoreductive surgery and HITHOC could improve local pleural control and overall survival for selected patients with advanced NSCLC confined in the chest seems based on a good rationale. It is therefore fascinating that our systematic review, on the possible role of debulking surgery and HITHOC for selected stage IV NSCLC, confirms that something "new" should and could be done to prolong survival. A mean survival of 27 months, with 13 out of 21 (62\%) patients alive at 1 year and 10 patients still alive at the time of the respective publication in the 21 patients included (table 1) are positive results for those who, on the basis of the present literature, have a poor prognosis, with a median overall survival of just 4 months, and 1- and 5-year survival rates of less than $16 \%$ and $2 \%$, respectively [18]. Although we did not look at perioperative complications, there was no systemic toxicity or treatment-related mortality.

Of note, all 21 patients included in our systematic review had adenocarcinoma of the lung and all of them had also been treated with chemotherapy. Certainly, the appropriate indications for this type of surgery are mandatory to obtain longer survival. Surgical indications in this group of patients could be proposed as summarised in table 2. The exclusion of N2 and N3 patients is mandatory, and therefore correct histology of mediastinal nodes prior to surgery is obligatory. In the case of PET-positive N2 or N3, the standard protocol must include EBUS and/or video-mediastinoscopy to guarantee that no patient is denied surgery because of a false-positive PET scan or enlarged nodes on CT [19].

In 2003, Shigemura et al. [9] published a pilot study on HITHOC and panpleuropneumonectomy for patients with advanced carcinomatous pleuritis caused by lung cancer. Surgery was performed after HITHOC. The mean survival time was 19 months. IşıK et al. [8] performed cytoreduction and HITHOC in 19 patients overall. The 1-year survival rate was $54.7 \%$ in the HITHOC group, while it was $0.6 \%$ and $0.8 \%$ in the two historical control groups $(\mathrm{p}<0.01$ and $\mathrm{p}<0.05$, respectively). Both of our patients who underwent this procedure were reported in 2015 [3], but have since died. The first died after 7 months

TABLE 2 Proposed indications for debulking surgery and hyperthermic intrathoracic chemotherapy for stage IVA (M1a) nonsmall cell lung cancer

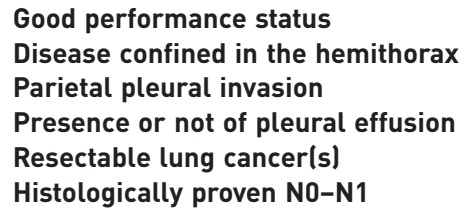




\begin{tabular}{|c|c|c|c|c|}
\hline First author [ref.] & Patients n & $\begin{array}{l}\text { Associated } \\
\text { procedure }\end{array}$ & $\begin{array}{l}\text { Median survival } \\
\text { time months }\end{array}$ & Survival rate \\
\hline KIMURA [20] & 7 & Debulking & & 0 deaths after 35 months \\
\hline Matsuzakı [21] & 11 & Debulking & 20 & \\
\hline $\mathrm{Hu}[22]$ & 54 & VATS pleural biopsy & 21.7 & $74.1 \%$ at 1 year \\
\hline YI [23] & 23 & Debulking & & $24.3 \%$ at 3 years \\
\hline Kodama [24] & 101 & Debulking & & $37.4 \%$ at 5 years \\
\hline
\end{tabular}

VATS: video-assisted thoracoscopic surgery.

because of sepsis following spinal surgery and the second died after 3 years because of tumour spread in the abdomen (the chest had minimal uptake on PET-CT).

Nevertheless, some other relevant publications not included in the systematic review (figure 1) confirmed a better survival of the study groups (table 3). Kimura et al. [20] demonstrated absence of death during a follow-up period of 9-35 months in seven patients who underwent HITHOC and debulking surgery for disseminated malignant pleural effusion for lung cancer. MATSUZAKi et al. [21] performed HITHOC following resection of the lung primary tumour (no pleurectomy) in 11 consecutive patients with primary pulmonary adenocarcinoma and pleural effusions containing tumour cells but without distant metastasis. The median survival time for the patients receiving perfusion treatment was 20 months, while the median survival time for the control group was only 6 months [16]. Hu et al. [22] performed video-assisted thoracoscopic surgery pleural biopsy and HITHOC in 54 patients with malignant pleural effusion caused by lung carcinoma with a median survival of 21.7 months and a 1-year survival rate of $74.1 \%$. YI et al. [23] performed an interesting study on 23 patients with advanced lung adenocarcinoma who underwent debulking surgery alone versus debulking surgery with HITHOC, which was continued for only $30 \mathrm{~min}$ (compared with $60 \mathrm{~min}$ in our technique). The complication rate was $34.8 \%$ versus $40 \%$, while the 3 -year survival rate was $24.3 \%$ versus $0 \%(\mathrm{p}=0.045)$, respectively. KoDAMA et al. [24] performed debulking surgery and HITHOC in 101 patients with pleural surface malignancy, obtaining a $37.4 \%$ survival rate at 5 years.

Of note, because all of the patients in our systematic review had adenocarcinoma, which presents high heterogeneity, other factors such as molecular biology indicators, classification and degree of infiltration could have had an impact on the therapeutic effect with a possible influence on long-term follow-up results [25]. Furthermore, on the basis of the survival obtained in our systematic review, which showed a mean survival of 27 months and a median survival of 18 months, we believe that there is a subgroup of lung cancer and pleural effusion/nodules confined in the chest that does not have the proper allocation in the tumour, node and metastasis classification. These tumours may be better downstaged to stage IIIB, and this is supported by the International Association for the Study of Lung Cancer Lung Cancer Staging Project that reported the survival for pathological stage IIIC and pathological stage IVA is about the same, with no statistically significant differences [26].

Furthermore, although the recent results of a prospective randomised study on the use of "solo" HITHOC or chemical talc pleurodesis in patients diagnosed with NSCLC and metastatic malignant pleural effusion have shown a dismal survival of only 8 months [27], the good results of our systematic review suggest that debulking surgery and HITHOC need to be investigated thoroughly with the intention to give hope, but not false hope, to the group of patients with advanced stage lung cancer.

In conclusion, although this systematic review shows that some encouraging evidence exists, the quality of evidence available concerning debulking surgery and HITHOC is still weak in order to confirm the effectiveness of this procedure for selected patients with advanced lung cancer. Therefore, better evidence, preferably in the form of randomised controlled trials, is necessary to evaluate clearly the benefit of this novel therapeutic approach. Setting standardised criteria for surgery is also mandatory. The positive results obtained in this systematic review can contribute to generating interest in this form of surgery for selected patients with stage IV lung cancer. This new interest could also drive further evidence supporting the prolongation of survival time while maintaining a good quality of life.

Acknowledgement: Part of this article was presented at the Fourth International Joint Meeting on Thoracic Surgery, Barcelona, November 28-30, 2018. The authors wish to thank Ramón Rami-Porta (Hospital Universitari Mútua Terrassa, Barcelona, Spain) and Laureano Molins (Hospital Clínic and Sagrat Cor, Barcelona, Spain) for their valuable comments and suggestions. 
Conflict of interest: None declared.

Support statement: This study has been funded by the University of Catania (Catania, Italy) within the FIR Research Program 2014-2016 and Department Research Program 2016-2018. Funding information for this article has been deposited with the Crossref Funder Registry.

\section{References}

Spiro SG, Silvestri GA. One hundred years of lung cancer. Am J Respir Crit Care Med 2005; 172: 523-529.

2 Migliore M, Calvo D, Criscione A, et al. Pleurectomy/decortication and hyperthermic intrapleural chemotherapy for malignant pleural mesothelioma: initial experience. Future Oncol 2015; 11: Suppl. 24, 19-22.

3 Migliore M, Calvo D, Criscione A, et al. Cytoreductive surgery and hyperthermic intrapleural chemotherapy for malignant pleural diseases: preliminary experience. Future Oncol 2015; 11: Suppl. 2, 47-52.

4 Li C, Juo SW, Hsu HH, et al. Lung adenocarcinoma with intraoperatively diagnosed pleural seeding: is main tumor resection beneficial for prognosis? J Thorac Cardiovasc Surg 2018; 155: 1238-1249.

5 Hanagiri T, Takenaka M, Oka S, et al. Results of a surgical resection for patients with stage IV non-small-cell lung cancer. Clin Lung Cancer 2012; 13: 220-224.

6 Iida T, Shiba M, Yoshino I, et al. Surgical intervention for non-small-cell lung cancer patients with pleural carcinomatosis: results from the Japanese Lung Cancer Registry in 2004. J Thorac Oncol 2015; 10: 1076-1082.

7 Zhou $\mathrm{H}, \mathrm{Wu} \mathrm{W}$, Tang X, et al. Effect of hyperthermic intrathoracic chemotherapy (HITHOC) on the malignant pleural effusion: a systematic review and meta-analysis. Medicine 2017; 96: e5532.

8 Işık AF, Şanlı M, Yılmaz M, et al. Intrapleural hyperthermic perfusion chemotherapy in subjects with metastatic pleural malignancies. Respir Med 2013; 107: 762-767.

9 Shigemura N, Akashi A, Ohta M, et al. Combined surgery of intrapleural perfusion hyperthermic chemotherapy and panpleuropneumonectomy for lung cancer with advanced pleural spread: a pilot study. Interact Cardiovasc Thorac Surg 2003; 2: 671-665.

10 Monneuse O, Beaujard AC, Guibert B, et al. Long-term results of intrathoracic chemohyperthermia (ITCH) for the treatment of pleural malignancies. Br J Cancer 2003; 88: 1839-1843.

11 Grile G. The effects of heat and radiation on cancers implanted on the feet of mice. Cancer Res 1963; 23: 372-380.

12 Barlogie B, Corry PM, Drewinko B. In vitro thermochemotherapy of human colon cancer cells with cisdichlorodiamineplatinium. Cancer Res 1980; 40: 1165-1168.

13 Carry Y, Brachet A, Gilly FN, et al. A new device for the treatment of pleural malignancies: intrapleural chemohyperthermia preliminary report. Oncology 1993; 50: 348-352.

14 Ried M, Potzger T, Braune N, et al. Cytoreductive surgery and hyperthermic intrathoracic chemotherapy perfusion for malignant pleural tumours: perioperative management and clinical experience. Eur J Cardiothorac Surg 2013 43: 801-807.

15 de Bree E, van Ruth S, Baas P, et al. Cytoreductive surgery and intraoperative hyperthermic intrathoracic chemotherapy in patients with malignant pleural mesothelioma or pleural metastases of thymoma. Chest 2002; 121: 480-487.

16 Ambrogi MC, Korasidis S, Lucchi M, et al. Pleural recurrence of thymoma: surgical resection followed by hyperthermic intrathoracic perfusion chemotherapy. Eur J Cardiothorac Surg 2016; 49: 321-326.

17 Zhang $\mathrm{H}$, Zhan $\mathrm{C}$, Ke J, et al. EGFR kinase domain mutation positive lung cancers are sensitive to intrapleural perfusion with hyperthermic chemotherapy (IPHC) complete treatment. Oncotarget 2016; 7: 3367-3378.

18 Cetin K, Ettinger DS, Hei Y, et al. Survival by histologic subtype in stage IV nonsmall cell lung cancer based on data from the Surveillance, Epidemiology and End Results Program. Clin Epidemiol 2011; 3: 139-148.

19 Migliore M, Nardini M, Rogers LJ, et al. A pragmatic view of the usefulness of video-mediastinoscopy in the modern era. J Vis Surg 2018; 4: 145.

20 Kimura M, Tojo T, Naito H, et al. Effects of a simple intraoperative intrathoracic hyperthermotherapy for lung cancer with malignant pleural effusion or dissemination. Interact Cardiovascular Thorac Surg 2010; 10: 568-571.

21 Matsuzaki Y, Edagawa M, Shimizu T, et al. Intrapleural hyperthermic perfusion with chemotherapy increases apoptosis in malignant pleuritis. Ann Thorac Surg 2004; 78: 1769-1772.

$22 \mathrm{Hu} \mathrm{R}$, Jiang $\mathrm{H}, \mathrm{Li} \mathrm{H}$, et al. Intrapleural perfusion thermo-chemotherapy for pleural effusion caused by lung carcinoma under VATS. J Thorac Dis 2017; 9: 1317-1321.

23 Yi E, Kim D, Cho S, et al. Clinical outcomes of cytoreductive surgery combined with intrapleural perfusion of hyperthermic chemotherapy in advanced lung adenocarcinoma with pleural dissemination. $J$ Thorac Dis 2016; 8: $1550-1560$

24 Kodama K, Higashiyama M, Okami J, et al. Cytoreductive surgery and post-operative heated pleural chemotherapy for the management of pleural surface malignancy. Int J Hyperthermia 2013; 29: 653-662.

25 Rami-Porta R, Asamura H, Goldstraw P. Predicting the prognosis of lung cancer: the evolution of tumor, node and metastasis in the molecular age - challenges and opportunities. Transl Lung Cancer Res 2015; 4: 415.

26 Goldstraw P, Chansky K, Crowley J, et al. The IASLC Lung Cancer Staging Project: proposals for the revision of the stage grouping in the forthcoming (8th) edition of the TNM classification of lung cancer. $J$ Thorac Oncol 2016; 11: 39-51.

27 Kleontas A, Sioga A, Pandria N, et al. Clinical factors affecting the survival of patients diagnosed with non-small cell lung cancer and metastatic malignant pleural effusion, treated with hyperthermic intrathoracic chemotherapy or chemical talc pleurodesis: a single center, prospective, randomized trial. J Thorac Dis 2019; 11: 1788-1798. 